\title{
MENINGIOMA - DIFFERENT LOCALIZATIONS AND PRESENTATIONS
}

\author{
Daniela Stefanescu', Vlad Claudiu Stefanescu ${ }^{1}$, Inimioara Mihaela Cojocaru, ${ }^{1,2}$ \\ ${ }^{1}$ Colentina Clinical Hospital, Bucharest, Romania \\ 2"Carol Davila" University of Medicine and Pharmacy, Bucharest, Romania
}

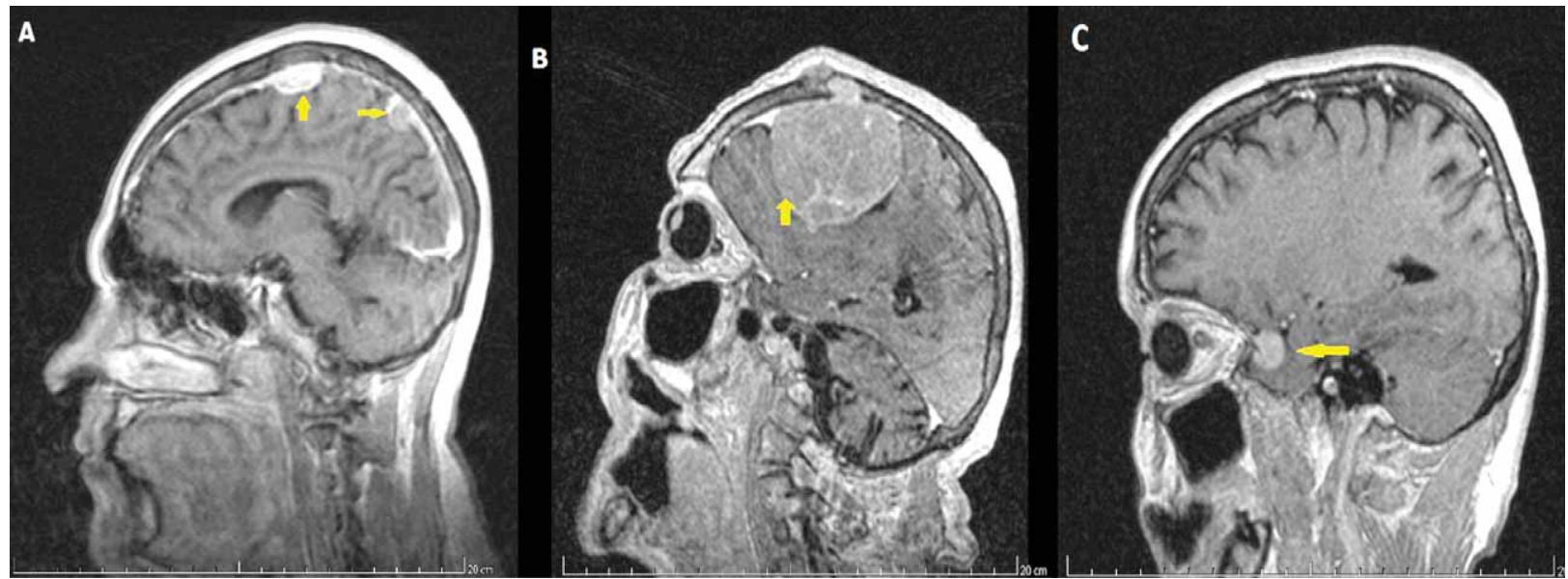

Depending on localization, the same type of expansive process may result in different clinical presentations. Such is the case of our patients. Patient A suffered her first focal motor seizure while sitting, which was briefly followed by loss of consciousness. Clinical examination revealed initial stupor, which reversed to fully conscious state, and by brisk involuntary clonoid movements involving her left side of her body, which were self-limited within several minutes. Brain MRI revealed frontal and parietal enhancing lesions protruding into the right hemisphere (arrows).

Patient B suffered from progressive muscle weakness involving her right limbs, and aphasia which started almost 6 months prior to her presentation in our Clinic. We performed an MRI which revealed a large $\mathrm{Gd}+$ mass in her left cerebral hemisphere with subfalciform herniation and frontal bone destruction.

The last patient presented into our clinic suffering only from diplopia which set on six weeks before. Neurologic examination did not find any other clinical sign apart from diplopia in the right lateral gaze. Brain MRI showed a solid mass of approximately $2 \mathrm{~cm}$ with calcifications situated adjacent to the cavernous sinus and sphenoid bone.

All lesions were well delineated from the surrounding parenchyma (in patient B also produced secondary mass effect on her hemispheres), and showed enhancement on Gadolinium administration, pointing to the diagnosis of meningioma.

Meningioma is a benign tumor of the meninges, which occur at sites of dural folds, most commonly the fronto-parietal parasagittal convexities, but also at the cerebello-pontine angle and on the sphenoid wing and the tuberculum sellae. They are highly vascularized, and are hypo/iso-intense on MRI and show enhancement. Clinical expression varies from asymptomatic to seizures and focal neurologic deficits. Treatment is surgical ${ }^{1}$.

${ }^{1}$ Intracranial Neoplasms and paraneoplastic disorders. In: Ropper A.H., Samuels M.A., Klein J.P. (eds.) Adams and Victors Principles of Neurology, Palatino: McGraw-Hill Education; 2014; p. 639-696 